\title{
Magnetic spin structure and magnetoelectric coupling in $\mathrm{BiFeO}_{3}-\mathrm{BaTiO}_{3}$ multilayer
}

Vera Lazenka, Michael Lorenz, Hiwa Modarresi, Manisha Bisht, Rudolf Rüffer, Michael Bonholzer, Marius Grundmann, Margriet J. Van Bael, André Vantomme, and Kristiaan Temst

Citation: Appl. Phys. Lett. 106, 082904 (2015); doi: 10.1063/1.4913444

View online: https://doi.org/10.1063/1.4913444

View Table of Contents: http://aip.scitation.org/toc/apl/106/8

Published by the American Institute of Physics

\section{Articles you may be interested in}

Correlation of magnetoelectric coupling in multiferroic $\mathrm{BaTiO}_{3}-\mathrm{BiFeO}_{3}$ superlattices with oxygen vacancies and antiphase octahedral rotations

Applied Physics Letters 106, 012905 (2015); 10.1063/1.4905343

Interface induced out-of-plane magnetic anisotropy in magnetoelectric $\mathrm{BiFeO}_{3}-\mathrm{BaTiO}_{3}$ superlattices

Applied Physics Letters 110, 092902 (2017); 10.1063/1.4977434

Giant magnetoelectric coupling interaction in $\mathrm{BaTiO}_{3} / \mathrm{BiFeO}_{3} / \mathrm{BaTiO}_{3}$ trilayer multiferroic heterostructures

Applied Physics Letters 107, 082908 (2015); 10.1063/1.4929729

Multiferroic magnetoelectric composites: Historical perspective, status, and future directions

Journal of Applied Physics 103, 031101 (2008); 10.1063/1.2836410

Multiferroic oxide thin films and heterostructures

Applied Physics Reviews 2, 021304 (2015); 10.1063/1.4921545

Electric field control of magnetism using $\mathrm{BiFeO}_{3}$-based heterostructures

Applied Physics Reviews 1, 021303 (2014); 10.1063/1.4870957

\section{Conference Proceedings}




\title{
Magnetic spin structure and magnetoelectric coupling in $\mathrm{BiFeO}_{3}-\mathrm{BaTiO}_{3}$ multilayer
}

\author{
Vera Lazenka, ${ }^{1, a)}$ Michael Lorenz, ${ }^{2}$ Hiwa Modarresi, ${ }^{1}$ Manisha Bisht, ${ }^{1}$ Rudolf Rüffer, ${ }^{3}$ \\ Michael Bonholzer, ${ }^{2}$ Marius Grundmann, ${ }^{2}$ Margriet J. Van Bael, ${ }^{4}$ André Vantomme, ${ }^{1}$ \\ and Kristiaan Temst ${ }^{1}$ \\ ${ }^{1}$ KU Leuven, Instituut voor Kern- en Stralingsfysica, Celestijnenlaan 200 D, 3001 Leuven, Belgium \\ ${ }^{2}$ Institut für Experimentelle Physik II, Universität Leipzig, Linnéstraße 5, D-04103 Leipzig, Germany \\ ${ }^{3}$ European Synchrotron Radiation Facility, CS 40220, 38043 Grenoble Cédex 9, France \\ ${ }^{4}$ Laboratorium voor Vaste-Stoffysica en Magnetisme, KU Leuven, Celestijnenlaan 200 D, 3001 Leuven, \\ Belgium
}

(Received 21 January 2015; accepted 11 February 2015; published online 23 February 2015)

\begin{abstract}
Magnetic spin structures in epitaxial $\mathrm{BiFeO}_{3}$ single layer and an epitaxial $\mathrm{BaTiO}_{3} / \mathrm{BiFeO}_{3}$ multilayer thin film have been studied by means of nuclear resonant scattering of synchrotron radiation. We demonstrate a spin reorientation in the $15 \times\left[\mathrm{BaTiO}_{3} / \mathrm{BiFeO}_{3}\right]$ multilayer compared to the single $\mathrm{BiFeO}_{3}$ thin film. Whereas in the $\mathrm{BiFeO}_{3}$ film, the net magnetic moment $\vec{m}$ lies in the (1-10) plane, identical to the bulk, $\vec{m}$ in the multilayer points to different polar and azimuthal directions. This spin reorientation indicates that strain and interfaces play a significant role in tuning the magnetic spin order. Furthermore, large difference in the magnetic field dependence of the magnetoelectric coefficient observed between the $\mathrm{BiFeO}_{3}$ single layer and multilayer can be associated with this magnetic spin reorientation. (C) 2015 AIP Publishing LLC.

[http://dx.doi.org/10.1063/1.4913444]
\end{abstract}

The coupling between magnetic and ferroelectric orders, known as magnetoelectric (ME) effect, ${ }^{1}$ attracts growing interest in science and technology due to the rich fundamental physics behind the phenomenon and its promising application potential. ${ }^{2,3}$ Control of magnetization without the need for a magnetic field offers possibilities for novel ME memory and spintronic devices ${ }^{1-4}$ with reduced energy consumption and higher speed. Many new multiferroic materials with coupled (anti-)ferromagnetic and ferroelectric orders have been discovered recently, ${ }^{5-7}$ however, there are very few systems with ordering temperatures above $300 \mathrm{~K}$. In most cases, these are $\mathrm{BiFeO}_{3}$-based multiferroics, in which the ferroelectric and antiferromagnetic transition temperatures are $\mathrm{T}_{\mathrm{C}} \sim 1150 \mathrm{~K}$ and $\mathrm{T}_{\mathrm{N}} \sim 640 \mathrm{~K}$, respectively. It has long been known that $\mathrm{BiFeO}_{3}$ has a G-type canted antiferromagnetic structure. A net magnetic moment arising from this canted spin structure is averaged out to zero due to the spin cycloid (62 $\pm 2 \mathrm{~nm}$ period) propagating along the [110] direction; its spin rotation plane is $(1-10)$, as established by neutron diffraction. ${ }^{8,9}$ Using the same technique, it has been demonstrated by Béa et al. ${ }^{10}$ that this cycloidal spiral modulation is suppressed by high epitaxial strain in thin films. Furthermore, the magnetic spin structure of $\mathrm{BiFeO}_{3}$ films is sensitive to minor changes in its crystal structure arising from a varying degree of strain relaxation, depending on the substrate material ${ }^{11}$ and orientation. ${ }^{12}$

Recently, ${ }^{7}$ we have demonstrated a remarkable magnetoelectric response in $\mathrm{BaTiO}_{3} / \mathrm{BiFeO}_{3}$ composite thin films and multilayers, where the value of the magnetoelectric coefficient $\alpha_{M E}$ reaches $20.75 \mathrm{~V} / \mathrm{cmOe}$, comparable to the highest value reported in literature. ${ }^{13} \mathrm{We}$ have also

\footnotetext{
${ }^{\text {a) }}$ Author to whom correspondence should be addressed. Electronic mail: Vera.Lazenka@fys.kuleuven.be
}

correlated the magnetoelectric coefficient of $\mathrm{BaTiO}_{3} /$ $\mathrm{BiFeO}_{3}$ multilayers grown at different oxygen partial pressures to oxygen vacancies and antiphase octahedral rotations. ${ }^{14}$ The saturation magnetization of the multilayer film consisting of $15 \times\left[\mathrm{BaTiO}_{3} / \mathrm{BiFeO}_{3}\right]$ is substantially larger than that of the single-phase $\mathrm{BaTiO}_{3}$ and $\mathrm{BiFeO}_{3}$ films. Additionally, it was shown by Toupet et al. ${ }^{15}$ that the magnetization in such multilayers increases with an increasing number of interfaces in the superlattice. As the magnetic properties of $\mathrm{BiFeO}_{3}$ are highly dependent on the changes in strain, a detailed study of the local magnetic structure in such multilayer films is critically important.

In this letter, on the one hand, we report on the determination of the local magnetic spin structure of a $\mathrm{BiFeO}_{3}$ film and a $15 \times\left[\mathrm{BaTiO}_{3} / \mathrm{BiFeO}_{3}\right]$ multilayer by means of nuclear resonant scattering (NRS) of synchrotron radiation and demonstrate that the $\mathrm{BiFeO}_{3}$ layer has different magnetic spin structures in the single film compared to the multilayer. On the other hand, the crystal structure and strain state in the films are studied using x-ray reciprocal space maps (RSMs). We demonstrate that the magnetoelectric response of these thin films strongly depends on the variations of strain and on the presence of interfaces. This local information helps to understand the relationship between the magnetic spin structure in $\mathrm{BiFeO}_{3}$ layers and strain-mediated magnetoelectric coupling in multilayers.

The $500 \mathrm{~nm}$-thick $\mathrm{BiFeO}_{3}$ thin film (referred to as the "thin film") and $15 \times\left[\mathrm{BaTiO}_{3} / \mathrm{BiFeO}_{3}\right]$ multilayer with the double layer thickness of $14 \mathrm{~nm}$ (referred to as the "multilayer") were grown by pulsed laser deposition (PLD) both on single-crystalline $\mathrm{SrTiO}_{3}$ (STO) (001) and $\mathrm{SrTiO}_{3}: 0.5 \% \mathrm{Nb}(001)$ substrates. A $\mathrm{KrF}$ excimer laser was used to grow films at a substrate temperature of $680^{\circ} \mathrm{C}$ and oxygen partial pressure of 0.25 mbar. For more details of the 
PLD growth, film structure, magnetic and ferroelectric response of the films, see Lorenz et al. ${ }^{7,14}$ The crystalline structure and elastic strain of the films used for NRS were characterized by X-ray diffraction (XRD) using a PANalytical X'pert PRO MRD with parabolic mirror $\left(\mathrm{Cu} \mathrm{K}{ }_{\alpha}\right.$ line focus) and a PIXcel ${ }^{3 \mathrm{D}}$ detector with variable channel number. Figure 1 shows RSMs around the symmetric (002) and asymmetric $(-103)$ STO substrate peaks, together with the weaker thin film and multilayer peaks, respectively. From the vertical alignment (i.e., close by $\mathrm{q}_{\|}[110]$ values) of the film and substrate peaks, the in-plane lattice match can be deduced. The $\mathrm{BiFeO}_{3}$ thin film grown on STO is nearly in-plane lattice matched, while the lack of vertical alignment of $(-103)$ film and substrate peaks in case of the multilayer indicates the relaxation of its lattice. The sharp peaks of the multilayer indicate its higher crystalline quality as opposed to the pronounced horizontal and vertical peak broadening in the $\mathrm{BiFeO}_{3}$ film due to higher tilt mosaicity and variation of lattice constants.

The superlattice periodicity creates multilayer satellite peaks, visible up to the fourth order. A superlattice period $L_{\mathrm{BIFeO} 3+\mathrm{BaTiO}_{3}}=(13.9 \pm 0.7) \mathrm{nm}$ is obtained from $L=\lambda /$ $\left(2 \Delta \theta \cdot \cos \theta_{B}\right)$, where $\lambda$ is the $\mathrm{X}$-ray wavelength, $\Delta \theta$ is the angular separation between two adjacent satellite peaks (i.e., vertical direction in the RSMs), and $\theta_{B}$ is the Bragg angle of the zero-order satellite peak. Consistent thickness values of $6.1 \mathrm{~nm}\left(\mathrm{BaTiO}_{3}\right)$ and $7.7 \mathrm{~nm}\left(\mathrm{BiFeO}_{3}\right)$ were obtained from scanning transmission electron microscopy cross sections ${ }^{14}$ in which an excellent stacking of $\mathrm{BaTiO}_{3}$ and $\mathrm{BiFeO}_{3}$ layers is evident.

The local magnetic spin structure in non ${ }^{57} \mathrm{Fe}$-enriched $\mathrm{BiFeO}_{3}$ layers was investigated at room temperature using NRS in forward scattering geometry, known as nuclear forward scattering (NFS), ${ }^{16}$ at the nuclear resonance beamline (ID18), European Synchrotron Radiation Facility (ESRF).
The interference patterns were collected in grazing incidence geometry at a fixed angle of $0.2^{\circ}$ (the beam direction $\vec{k}$ is indicated in Figs. 2(c) and 2(d)). A high-resolution monochromator with $1 \mathrm{meV}$ bandwidth was tuned to the nuclear resonance energy $14.413 \mathrm{keV}$ of the Mössbauer (MB) transition in ${ }^{57} \mathrm{Fe}$. Because of the large bandwidth compared to typical hyperfine splittings $(\sim 10 \mathrm{neV})$, the synchrotron $\mathrm{X}$-ray beam impinging on the sample coherently scatters in forward direction via excitation of all hyperfine nuclear levels with the characteristic lifetime of the ${ }^{57} \mathrm{Fe} \mathrm{MB}$ transition (141 ns). The delayed reemitted photons are collected as a function of time and as a result of the minor relative differences in their energy $\left(\sim 10^{-12} \mathrm{eV}\right)$, an interference pattern is obtained (see Fig. 2), which reveals at the atomic level the magnetic moment structure and chemical environment. Since, in case of ${ }^{57} \mathrm{Fe}$, the hyperfine field is antiparallel to the magnetic moment of the atoms, the NFS time spectra contain direct information about the orientation of the Fe magnetic moment, which is not available through conventional Mössbauer spectroscopy.

The measured time decay spectra for the thin film and the multilayer are shown in Figs. 2(a) and 2(b), clearly revealing that the beat patterns are different for the multilayer and the thin film. To extract the chemical and magnetic structure of $\mathrm{BiFeO}_{3}$ layers, a model was applied to the measured time spectra. The fittings (also shown in Figs. 2(a) and 2(b)) were done based on the dynamical theory of nuclear resonant scattering with the program package CONUSS. ${ }^{16,17}$ In this model, the polar angle $\theta$ is defined as the angle between the sample normal, i.e., the [001] crystallographic direction and the magnetic moment $\vec{m}$, see Figs. 2(c) and 2(d). The azimuthal angle $\varphi$ defines the in-plane rotation of the magnetic moment, i.e., the angle of the $\vec{m}$ projection in the sample plane with respect to the [110] direction.

The same model was applied to fit both spectra. During the fitting, two types of chemical states (sites) of Fe were
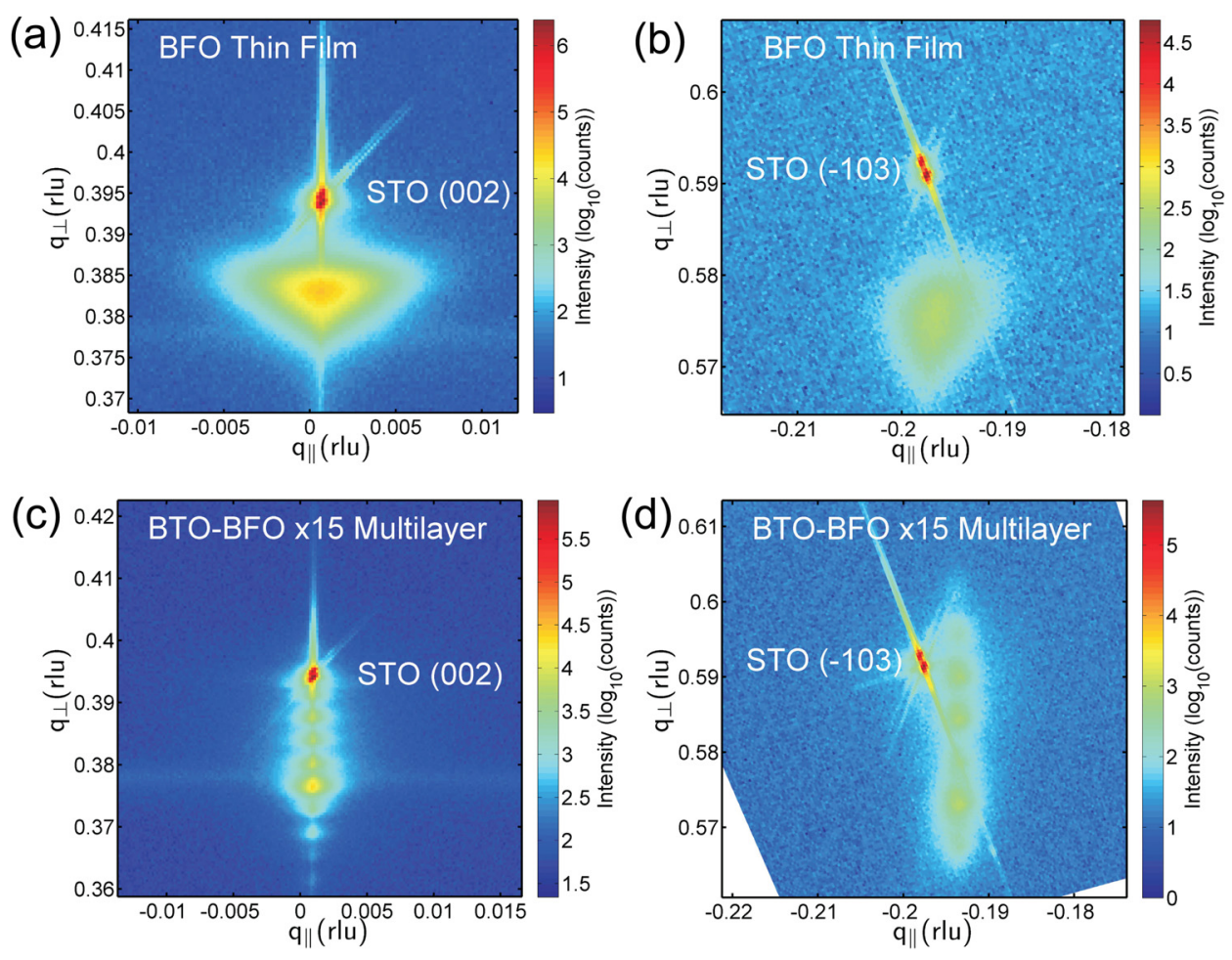

FIG. 1. XRD reciprocal space maps around symmetric (002) (a) and (c), and asymmetric (-103) (b) and (d) reflections of typical $\mathrm{BiFeO}_{3}$ film and $15 \times\left[\mathrm{BaTiO}_{3} / \mathrm{BiFeO}_{3}\right]$ multilayer samples, as indicated. The $\mathrm{BiFeO}_{3}$ film is grown in-plane lattice matched, as the vertical alignment of film and STO $(-103)$ peaks in (b) show. The RSMs of the multilayer (c) and (d) show superlattice peaks. BFO stands for $\mathrm{BiFeO}_{3}$ and $\mathrm{BTO}$ for $\mathrm{BaTiO}_{3}$. 
(a)

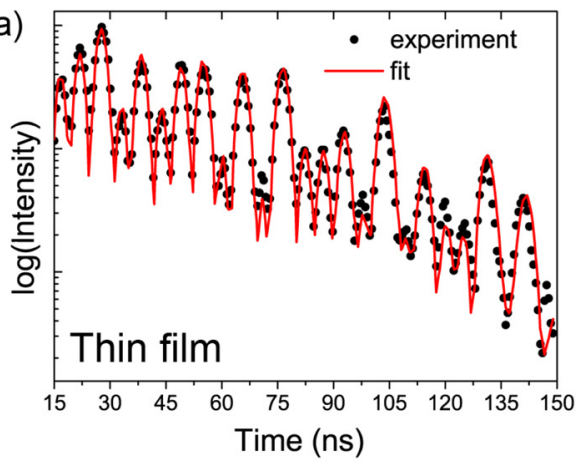

(b)

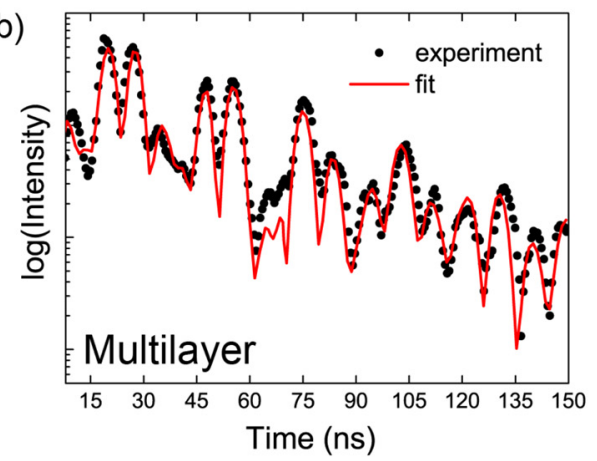

(c)

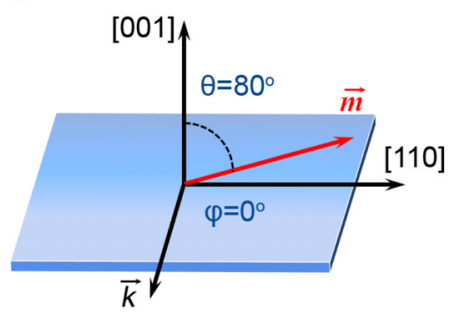

(d)

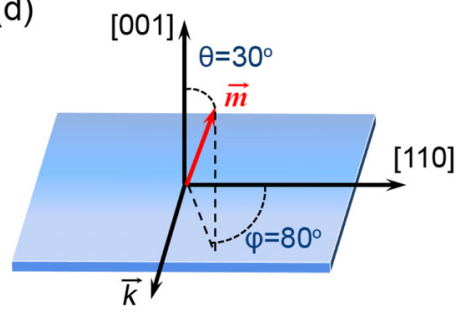

FIG. 2. (a) NFS time spectra of the $\mathrm{BiFeO}_{3}$ thin film and (b) $15 \times\left[\mathrm{BaTiO}_{3} /\right.$ $\left.\mathrm{BiFeO}_{3}\right]$ multilayer. The spectra were recorded at room temperature and zero external magnetic field. The solid lines are the model fits of the spectra. (c) and (d) Schematical representation of the fitting model for (c) the thin film and (d) the multilayer. $\vec{m}$ is the magnetic moment of the $\mathrm{BiFeO}_{3}$ layer(s) and $\vec{k}$ is the wave vector along the photon beam. taken into account. The main site, appearing as a single component, is attributed to the $\mathrm{BiFeO}_{3}$ phase with hyperfine fields of $49 \mathrm{~T}$ and $49.5 \mathrm{~T}$ and isomer shift values of $0.4 \mathrm{~mm} / \mathrm{s}$ and $0.33 \mathrm{~mm} / \mathrm{s}$ for the thin film and the multilayer, respectively. The second site with zero isomer shift and a smaller hyperfine field of $24 \mathrm{~T}$ is ascribed to the magnetic Fe site formed in the proximity of the surface and/or interface due to the breaking of the crystal symmetry in $\mathrm{BiFeO}_{3}$. The isomer shift values are given relative to the second Fe site. As the number of interfaces increases for the multilayer, the fraction of this site increases from 5\% in the thin film to $15 \%$ in the multilayer. The isomer shift reflects the electron density in the vicinity of the ${ }^{57} \mathrm{Fe}$ nucleus, and therefore the valence states of $\mathrm{Fe}$ in the films. From the similarity in the hyperfine fields and isomer shifts values associated with the different $\mathrm{Fe}$ sites in the film and multilayer, we can conclude that there is no substantial difference in the electronic configurations of these Fe atoms. Furthermore, based on the hyperfine parameters, a valence state of $\mathrm{Fe}^{3+}$ was determined both in the thin film and multilayer. These values of hyperfine parameters are in good agreement with conventional Mössbauer studies performed by Lebeugle et al., ${ }^{9}$ Sando et al., ${ }^{11}$ Tanaka et al. ${ }^{18}$ and Prado-Gonjal et al. ${ }^{19}$

The only parameters which differ between the thin film and the multilayer are associated with the orientation of the magnetic moment, i.e., the $\theta$ and $\varphi$ angles. Figures 2(c) and 2(d) show the orientation of the magnetic moment in the single film and multilayer, respectively. In the thin film, the in-plane angle $\varphi=0^{\circ}$, implying that $\vec{m}$ lies in the (1-10) plane, i.e., the plane of spin-rotation in bulk $\mathrm{BiFeO}_{3}$ according to Lebeugle et al. ${ }^{9}$ However, a spin reorientation occurs in the multilayer, see Fig. 2(d), and the magnetic moment does not belong to the spiral (1-10) plane, which might be an indication of the spin spiral suppression. The results are in line with the work of Sando et al., ${ }^{11}$ where it is shown that the bulk-like spiral spin structure in $\mathrm{BiFeO}_{3}$ films is suppressed when the lattice mismatch between $\mathrm{BiFeO}_{3}$ and substrate exceeds $1 \%$ Moreover, it has been demonstrated ${ }^{20,21}$ that $\mathrm{BiFeO}_{3}$ films grown on STO substrate are pseudomorphic (fully strained) when their thickness is less than $30 \mathrm{~nm}$. As the film thickness reaches $1 \mu \mathrm{m}$, the lattice parameter gradually approaches the bulk $\mathrm{BiFeO}_{3}$ value. ${ }^{21}$ Hence, in highly strained $\mathrm{BiFeO}_{3}$ layers within the multilayer, the spiral structure seems to be suppressed.

Previously, ${ }^{7}$ we have found that the saturation magnetization of the multilayer is substantially larger than that of the single-phase $\mathrm{BiFeO}_{3}$ and $\mathrm{BaTiO}_{3}$. Hence, the origin of the enhanced magnetization can be the suppression of the spiral spin magnetic structure due to high epitaxial strain in the $\mathrm{BiFeO}_{3}$ layers, which has been debated in the literature since the work of Wang et al. ${ }^{22}$ Moreover, as both $\mathrm{BiFeO}_{3}$ and $\mathrm{BaTiO}_{3}$ thin films possess weak net magnetic moments, ${ }^{7}$ a magnetic interaction between these layers at the interface may also contribute to the increased magnetic moment in the multilayers in comparison with the single-phase films.

The effect of strain and interfaces on the magnetoelectric coupling in $\mathrm{BiFeO}_{3}$ was studied for a number of thin films and multilayers. To this end, a direct longitudinal AC method was used to measure the ME coefficient $\alpha_{M E}$ as a function of static magnetic field. For details of this method, see Refs. 7 and 23. From Fig. 3(a), it is clear that $\alpha_{M E}$ of the multilayer is notably larger than that of the thin film. In addition, the temperature dependence (not shown here) and the magnetic field dependence of their ME response differ. While for the multilayer, the ME coefficient increases with magnetic field and saturates around $4 \mathrm{~T}, \alpha_{M E}$ for the $\mathrm{BiFeO}_{3}$ film reaches its maximum at $0.5 \mathrm{~T}$ with a subsequent decrease. The reason behind these trends is still unclear and under investigation. It is worth noting that the $\alpha_{M E}$ value and its behavior in DC magnetic field for the single-phase $\mathrm{BiFeO}_{3}$ thin film resemble those of $\mathrm{BiFeO}_{3} / \mathrm{BaTiO}_{3}$ composite films grown from mixed phase PLD targets. ${ }^{7}$ Since the 
(a)

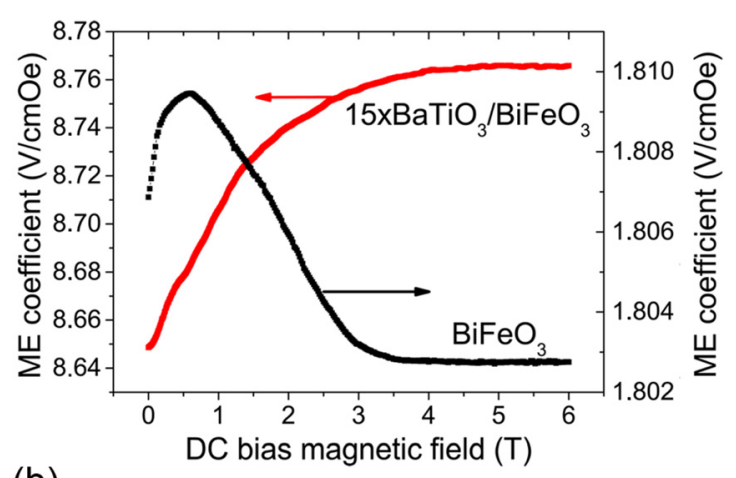

(b)

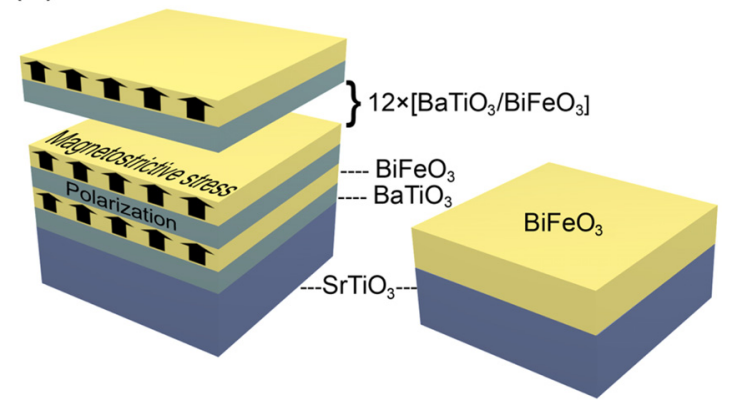

FIG. 3. (a) ME coefficient $\alpha_{M E}$ as a function of DC magnetic field (at $300 \mathrm{~K}$ ) for the $15 \times\left[\mathrm{BaTiO}_{3} / \mathrm{BiFeO}_{3}\right]$ multilayer and the $\mathrm{BiFeO}_{3}$ thin film. (b) Schematic of a ferroelectric-multiferroic multilayer (left) showing the development of the ME effect through magnetostrictive-piezoelectric interface coupling (arrows represent magnetostrictive stress); and a $\mathrm{BiFeO}_{3}$ thin film (right).

multilayer additionally contains piezoelectric/piezomagnetic interfaces, an extra ME coupling in multilayers may occur via the horizontal interfaces through strain-mediated interface coupling. In this case, upon application of an external magnetic field, magnetostrictive stress is produced in the weak ferromagnetic $\mathrm{BiFeO}_{3}$ layer and is transferred to ferroelectric $\mathrm{BaTiO}_{3}$ layer through the interface, see Fig. 3(b). This mechanical stress generates an electric potential difference in the ferroelectric layer via a piezoelectric effect. ${ }^{24}$ Evidently, two main factors affect ME coupling in the multilayer compared to the $\mathrm{BiFeO}_{3}$ single layer: suppression of the spiral spin structure in highly strained $\mathrm{BiFeO}_{3}$ layers observed through reorientation and increase of the net magnetic moment, and strain-mediated interface coupling via ferroelectric/multiferroic interfaces.

In conclusion, the local magnetic spin structure of a $\mathrm{BiFeO}_{3}$ single film and a $15 \times\left[\mathrm{BaTiO}_{3} / \mathrm{BiFeO}_{3}\right]$ multilayer grown epitaxially on $\mathrm{SrTiO}_{3}$ substrates have been studied using nuclear resonant forward scattering. While the electronic configuration of the $\mathrm{Fe}$ atoms does not undergo substantial changes, a spin reorientation occurs in the highly strained $\mathrm{BiFeO}_{3}$ layers within the multilayer compared to the single film. Furthermore, we correlate the enhanced magnetic and magnetoelectric properties observed in the multilayers with the suppression of the spiral spin magnetic structure, which is due to high epitaxial strain in the multilayer and interfacial interaction between ferroelectric
$\mathrm{BaTiO}_{3}$ and multiferroic $\mathrm{BiFeO}_{3}$. These multilayer heterostructures, consisting of multiferroic and ferroelectric layers, have promising perspectives due to their tailored properties (through epitaxial strain and interfacial coupling) and are good candidates for advanced devices with tunable magnetoelectric functionalities.

We thank Gabriele Ramm for PLD target preparation, Holger Hochmuth for PLD film growth, and Michael Bonholzer for the RSM presentation software. The authors gratefully acknowledge financial support from the Research Foundation Flanders (FWO), the Concerted Research Actions GOA/09/006 and GOA/14/007, and of the Deutsche Forschungsgemeinschaft (DFG) within SFB 762 "Functionality of oxide interfaces."

${ }^{1}$ M. Fiebig, J. Phys. D: Appl. Phys. 38, R123 (2005).

${ }^{2}$ L. W. Martin and R. Ramesh, Acta Mater. 60, 2449 (2012).

${ }^{3}$ H. Béa, M. Gajek, M. Bibes, and A. Barthélémy, J. Phys.: Condens. Matter 20, 434221 (2008).

${ }^{4}$ M. Bibes and A. Barthélémy, Nat. Mater. 7, 425 (2008).

${ }^{5}$ L. V. Costa, M. G. Ranieri, M. Cilense, E. Longo, and A. Z. Simões, J. Appl. Phys. 115, 17D910 (2014).

${ }^{6}$ T.-T. Liu, Z. Wang, J. Mao, N.-N. Cheng, and L.-J. Ni, J. Appl. Phys. 115, 17 C729 (2014).

${ }^{7}$ M. Lorenz, V. Lazenka, P. Schwinkendorf, F. Bern, M. Ziese, H. Modarresi, A. Volodin, M. J. Van Bael, K. Temst, A. Vantomme, and M. Grundmann, J. Phys. D: Appl. Phys. 47, 135303 (2014).

${ }^{8}$ I. Sosnowska, T. Peterlin-Neumaier, and E. Steichele, J. Phys. C 15, 4835 (1982).

${ }^{9}$ D. Lebeugle, D. Colson, A. Forget, M. Viret, P. Bonville, J. F. Marucco, and S. Fusil, Phys. Rev. B 76, 024116 (2007).

${ }^{10}$ H. Béa, M. Bibes, S. Petit, J. Kreisel, and A. Barthélémy, Philos. Mag. Lett. 87, 165 (2007).

${ }^{11}$ D. Sando, A. Agbelele, D. Rahmedov, J. Liu, P. Rovillain, C. Toulouse, I. C. Infante, A. P. Pyatakov, S. Fusil, E. Jacquet, C. Carrétéro, C. Deranlot, S. Lisenkov, D. Wang, J.-M. Le Breton, M. Cazayous, A. Sacuto, J. Juraszek, A. K. Zvezdin, L. Bellaiche, B. Dkhil, A. Barthélémy, and M. Bibes, Nat. Mater. 12, 641 (2013).

${ }^{12}$ W. Ratcliff, D. Kan, W. Chen, S. Watson, S. Chi, R. Erwin, and I. Takeuchi, Adv. Funct. Mater. 21, 1567 (2011).

${ }^{13}$ S. Dong, J. Zhai, J. Li, and D. Viehland, Appl. Phys. Lett. 89, 252904 (2006).

${ }^{14}$ M. Lorenz, G. Wagner, V. Lazenka, P. Schwinkendorf, H. Modarresi, M. J. Van Bael, A. Vantomme, K. Temst, O. Oeckler, and M. Grundmann, Appl. Phys. Lett. 106, 012905 (2015).

${ }^{15}$ H. Toupet, V. V. Shvartsman, F. Lemarrec, P. Borisov, W. Kleemann, and M. Karkut, Integr. Ferroelectr. 100, 165 (2008).

${ }^{16}$ R. Röhlsberger, Nuclear Condensed Matter Physics with Synchrotron Radiation-Basic Principles, Methodology and Applications, Springer Tracts in Modern Physics Vol. 208 (Springer Publishers, 2004).

${ }^{17}$ W. Sturhahn, Hyperfine Interact. 125, 149 (2000).

${ }^{18}$ K. Tanaka, Y. Tsukamoto, S. Okamura, and Y. Yoshida, Key Eng. Mater. 582, 63 (2013).

${ }^{19}$ J. Prado-Gonjal, D. Ávila, M. E. Villafuerte-Castrejón, F. GonzálezGarcía, L. Fuentes, R. W. Gómez, and E. Morán, Solid State Sci. 13, 2030 (2011).

${ }^{20}$ Y. H. Chu, T. Zhao, M. P. Cruz, Q. Zhan, P. L. Yang, L. W. Martin, and R. Ramesh, Appl. Phys. Lett. 90, 252906 (2007).

${ }^{21}$ M. B. Holcomb, L. W. Martin, A. Scholl, Q. He, P. Yu, C.-H. Yang, and R. Ramesh, Phys. Rev. B 81, 134406 (2010).

${ }^{22}$ J. Wang, J. B. Neaton, H. Zheng, V. Nagarajan, S. B. Ogale, B. Liu, and R. Ramesh, Science 299, 1719 (2003).

${ }^{23}$ V. V. Lazenka, G. Zhang, J. Vanacken, I. I. Makoed, A. F. Ravinski, and V. V. Moshchalkov, J. Phys. D: Appl. Phys. 45, 125002 (2012).

${ }^{24}$ M. Bichurin, V. Petrov, and G. Srinivasan, Phys. Rev. B 68, 054402 (2003). 